\title{
Recoding as a function of chunking and meaningfulness ${ }^{1,2}$
}

Trigrams of high or low meaningfulness were paired with 1-chunk or 2-chunk recoding cues in short-term memory. The presence or either 1-chunk or 2-chunk cues reduced but did not eliminate the effects of meaningfulness on trigram recall.

When recoding cues are paired with trigrams (e.g., Zero-ZER) in short-term memory (STM), the recoding cues may aid STM by reducing the number of chunks of information to be stored in and retrieved from memory. Lindley (1965) found that low meaningfulness (LM) trigrams were recalled as well as high meaningfulness (HM) trigrams when recoding cues which enabled the trigrams to be recoded into single chunks of information were present.

The present study investigated the effects of 1- and 2-chunk recoding cues on STM for LM and HM items. (REH-Rehearse is a 1-chunk trigram-recoding cue pair; REH-Relent a 2-chunk pair.) If STM is a function of number of chunks, then recall should depend upon the number of chunks of information in the trigramrecoding cue pair and not upon the level of meaningfulness of the items.

Method

The 2 by 3 factorial design had a between-Ss factor of 2 levels of trigram meaningfulness (low, high) and a within-Ss factor of 3 modes of trigram presentation (2 trigrams spelled twice (e.g., TUM TUM GAR GAR), 2 trigrams plus 1-chunk cues (e.g., TUM Tumble GAR Garden), 2 trigrams plus 2-chunk cues (e.g., TUM Tuft GAR Gallant)). For the 1-chunk cues, the first 3 letters of the cue always were the same as the trigram while for the 2-chunk cues, the first 2 letters of the cue always were the same as the trigram and the third letter was different.

The items were presented in 3 blocks of 6 items each with each block corresponding to one of the mode of trigram presentation conditions; the appropriate recoding cue-trigram rules were carefully explained to $S$. When recoding cues were presented, $S$ recalled both the trigram and the cue. Over Ss each block of items appeared equally often as the first, second, and third set of items recalled. Within blocks, the items were counterbalanced over serial positions over Ss. The mean Noble (1961) association values of the 36 LM CVC trigrams was $62 \%$; of the $36 \mathrm{HM} \mathrm{CVC}$ trigrams, $96 \%$.

$\mathrm{E}$ presented the items aurally to individual Ss in time to a $96 \mathrm{beats} / \mathrm{min}$. metronome. Seventy-two men and women college students were assigned randomly (with correction for equal Ns) to either the LM or HM trigram condition. E said "Ready"; paused for 3 beats, presented the to-be-recalled item; and then said a 3 -digit number. $S$ repeated the number and subtracted backwards by $3 \mathrm{~s}$ for 26 sec., when $\mathrm{E}$ said "Recall." S had $20 \mathrm{sec}$. to recall the items and then had a 5-sec. rest. Recall latencies (i.e., the time intervals between the recall cue and the onset of S's response) were measured by $\mathrm{E}$ with an electric timer. During the presentation of the items, E paused for 1 beat between each set of 3 letters and between each word. By such pauses and by his enunciation, $\mathrm{E}$ showed that each trigram was a unit.

\section{Results and Discussion}

Trigram Recall. The order in which the trigrams were recalled was ignored. Credit was given for each letter correctly recalled at the appropriate serial position (e.g., for item TUM GAR, the response TUM GAL or GAL TUM would be scored as 5 letters correct). The correct recall data for the trigrams are shown in the left-hand portion of Fig. 1. The HM trigrams were recalled better than the LM trigrams $(F=15.06$, df $=$ $1 / 70, p<.01)$. Mode of trigram presentation was highly significant $(F=75.71, d f=2 / 140, p<.01)$; the 2 -chunk condition produced poorer recall than the spelling condition while, as expected, the 1-chunk condition produced superior recall. As predicted, the 1-chunk cues produced better recall than the 2-chunk cues although a difference of this magnitude was not expected.

A major hypothesis under test here is that, when recoding cues are used, recall depends upon the number of chunks of information in the trigram-recoding cue pairs and not upon the meaningfulness (e.g., association

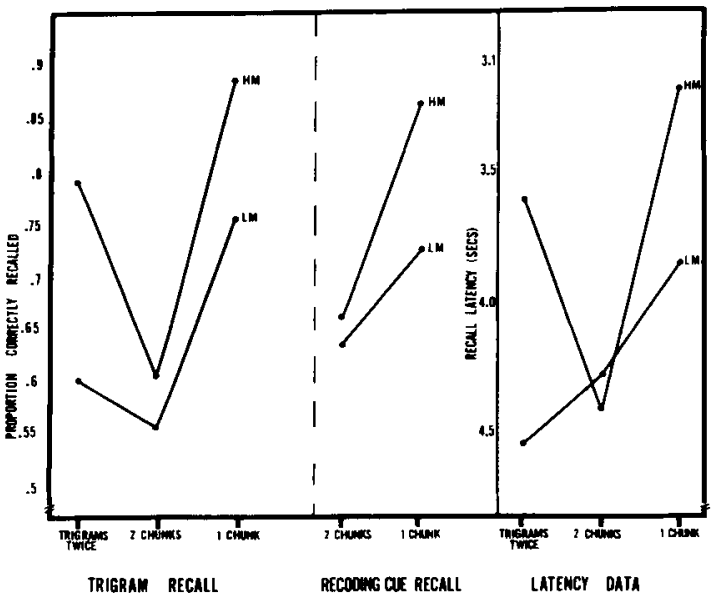

Fig. 1. Correct recall and latency data. 
values) of the trigrams. This hypothesis predicts a significant interaction between Meaningfulness and Mode of Trigram Presentation such that HM trigrams will be better recalled than LM trigrams when recoding cues are absent but that, when recoding cues are present, the LM 1-chunk pairs will be recalled as well as the HM 1-chunk pairs and the LM 2-chunk pairs will be recalled as well as the HM 2-chunk pairs. Inspection of Fig. 1 shows that the hypothesis was only partially confirmed; while there was a significant interaction between Meaningfulness and Mode of Trigram Presentation $(F=4.92, d f=2 / 140, p=.01)$, recall of HM trigrams was better than recall of LM trigrams bath with 1-chunk cues and with 2-chunk cues. The failure of these recall scores to support the hypothesis completely should be considered in context with the recoding cue recall scores.

Latency data. The latency data are presented in the right-hand portion of Fig. 1 (N. B., in order to facilitate comparisons of the latency and correct response data, the latencies are displayed in descending order on the ordinate). The latency data correspond closely to the correct recall data; the presence of 1-chunk cues produces faster responding than the 2-chunk conditions.

Recoding cue recall data. The correct recall data of the recoding cues are presented in the middle portion of Fig. 1. An analysis of variance showed that cues paired with HM trigrams were recalled better than cues paired with LM trigrams $(F=4.60, \mathrm{df}=1 / 170$, $\mathrm{p}<.05)$; thus at recall the recoding cues for the $\mathrm{HM}$ trigrams were more available as memory aids than were the cues for the LM trigrams. Therefore, part of the superior recall of HM trigrams over LM tri- grams with 1-chunk and with 2-chunk cues is due to the greater availability of the cues for the HM trigrams. In fact, the curves in Fig. 1 show a substantial relationship between recoding cue availability and trigram recall since when the difference in cue availability is small (HM and LM trigrams with 2-chunk cues), the difference in HM and LM trigram recall is small and when the difference in cue availability is larger (HM and LM trigrams with 1-chunk cues), the difference in $\mathrm{HM}$ and LM trigram recall is larger. While the present results are not definitive, they suggest that the efficacy of recoding cues depends upon the ability of such cues to reduce the number of chunks of information in the trigram-cue pair.

The 1-chunk cues were recalled better than the 2chunk cues $(F=37.73, d f=1 / 70, p<.01)$ showing that, as the memory load is increased in the 2-chunk conditions, recall falls off for recoding cues as well as for the trigrams. Finally, there was a significant Recoding Cue by Meaningfulness interaction ( $F=5.65$, $\mathrm{df}=1 / 70, \mathrm{p}<.05$ ) due to the bigger difference in recall of the 1-chunk and 2-chunk cues with high $M$ than with low $\mathrm{M}$ trigrams.

\section{References}

Lindley, R. H. Effects of trigram-recoding cue complexity on shortterm memory. J. verbal Learn, verbal Behav., 1965, 4, 274-279. Noble, C. E. Measurements of association value $(a)$, rated associations $\left(a^{\prime}\right)$ and scaled meaningfulness $\left(m^{\prime}\right)$ for the $2100 \mathrm{CVC}$ combinations of the English alphabet. Psychol. Rep., 1961, 8, 487521

\section{Notes}

1. Supported by a National Science Foundation research grant. 2. I wish to thank Daniel Edwards and Bernard Ostrowski for collecting the data. 